\title{
Análise de risco em ambiente hospitalar: uma proposta de trabalho
}

\author{
Carlos Alexandre Rodrigues Pereira \\ Graduado em Engenharia Ambiental pela Universidade de Uberaba, Uberaba/MG. Mestrando em Saúde \\ Pública e Meio Ambiente na Escola Nacional de Saúde Pública, Fundação Oswaldo Cruz. \\ Email:carlos.rpereira@hotmail.com
}

Lívia Caroline Souza Rezende.

Graduado em Engenharia Ambiental na Universidade de Uberaba, Uberaba/MG. Consultora Ambiental. Email: liviarezende.engambiental@hotmail.com

\section{Anselmo Ferreira da Silva}

Graduado em Engenharia Ambiental na Universidade de Uberaba, Uberaba/MG. Consultor em Engenharia Ambiental.

Email: anselmoferreira_22@hotmail.com

\section{Ana Maria Silva Siega}

Graduada em Engenharia Ambiental pela Universidade de Uberaba, Uberaba/MG.

Email:anasiega@yahoo.com.br

\section{Leandro de Oliveira Silva}

Graduado em Engenharia de Produção pela Universidade de Uberaba, Uberaba/MG. Docente dos cursos de Engenharia Ambiental, Química e Produção da Universidade de Uberaba-MG.

Email: leandro.oliveira@uniube.br

RESUMO: Nos hospitais, uma série de fatores relacionados à gestão pode facilitar a ocorrência de infecções hospitalares, como planos de gerenciamentos de resíduos inadequados, monitoramento da qualidade da água deficiente, falta de controle de vigilância sanitária e epidemiológica e falta da efetividade da gestão ambiental hospitalar. Esta pesquisa propõe metodologias de gestão para que os hospitais estejam em condições ambientais e sanitárias para a reabilitação das funções, capacidades e condições de saúde de seus usuários, no alcance da eficácia do atendimento oferecido pelas Instituições. A proposta envolve aplicações dos fundamentos e técnicas da Engenharia Ambiental, buscando a melhoria das condições de saneamento e saúde, por meio do levantamento e avaliação dos riscos inerentes ao ambiente hospitalar e formulação de propostas de controle. A metodologia utilizada consiste na visita a três hospitais de médio porte no Triângulo Mineiro/MG, com o objetivo de apontar os riscos relacionados à infecção hospitalar. Foi realizada a análise qualitativa dos dados coletados utilizando ferramentas de análise de riscos e qualidade, como o Braisntorming, HAZOP (Hazard and Operability Studies), Diagrama de Causa e Efeito e 5W2H. Por meio da aplicação das ferramentas de análise, foi possível propor ações simples e de baixo custo para a gestão dos riscos verificados, que podem ser aplicadas a qualquer hospital. As propostas conduzem a Gestão Ambiental por serem importantes meios para a identificação, diagnóstico, tratamento e monitoramento dos fatores de riscos ambientais ligados à atividade. Por meio deste trabalho propõe-se a atuação interdisciplinar em ambientes hospitalares para o desenvolvimento de técnicas e métodos adequados ao ambiente hospitalar.

PALAVRAS ChAVE: Análise de Risco, Gerenciamento de Risco, Fatores de Riscos.

\section{Risk analysis in hospital environment: a work proposal}

ABSTRACT; In hospitals, a number of factors related to management can facilitate the occurrence of nosocomial infections, such as waste management plans, inadequate monitoring of water quality poor and lack of control of sanitary and epidemiologic surveillance. This research proposes methods of management so that hospitals are in environmental and sanitary conditions for the rehabilitation of the functions, capabilities and health of its users, the extent of effectiveness of care provided by institutions. The proposal involves applications of the principles and techniques of Environmental Engineering, seeking to improve sanitation conditions and health, through the survey and assessment of risks inherent to the hospital environment and formulation of proposals for control. The methodology consists of visits to three hospitals of medium size in the Triângulo Mineiro/MG, with the aim of pointing out the risks related to hospital infection. We performed a qualitative analysis of data collected using tools of risk analysis and quality, as Braisntorming, HAZOP (Hazard and Operability Studies), Cause and Effect Diagram and $5 \mathrm{~W} 2 \mathrm{H}$. By applying these analysis tools, it was possible to propose simple and low cost actions for managing the risks found, which can be applied to any hospital. Proposals leading to environmental management to be important means for the identification, diagnosis, treatment and monitoring of environmental risk factors associated with the activity. Through this study proposes to interdisciplinary work in hospitals for the development of techniques and methods appropriate to the hospital environment.

Key Words: Risk Analysis, Risk Management, Risk Factors. 


\section{INTRODUÇÃO}

Para que os hospitais cumpram seu papel de reabilitação das funções, capacidades e condição de saúde de seus usuários é preciso que ele esteja em condições ambientais adequadas. Para a realização dos atendimentos sem que estes exponham os usuários nem às equipes de trabalho a riscos de contrair patologias, é necessário controle efetivo de fatores físicos, químicos e biológicos que podem causar doenças ou proliferar vetores.

Esses fatores, se não controlados, favorecem a ocorrência do principal risco no ambiente hospitalar: a Infecção Hospitalar.

No Brasil, o único estudo oficial sobre taxas de infecções nos hospitais brasileiros é limitado. Apesar das limitações de amostra (99 hospitais) e outros aspectos relacionados à coleta de dados é o único estudo nacional recente. Neste estudo, as infecções respiratórias inferiores correspondiam à $28,9 \%$, cirúrgicas $15,6 \%$, pele $15,5 \%$, urinárias $11 \%$ e sepses $10 \%$. Outras infecções apareceram em $18 \%$ das ocorrências (CONTROLE DE INCIDENTES HUMANOS - CIH, 2011).

Um estudo de prevalência realizado pelo Ministério da Saúde em 1994 revelou uma taxa média de infecção hospitalar de 14\%. Significa que, de 100 pessoas internadas quando da pesquisa, 14 apresentavam algum tipo de infecção contraída no hospital (CONTROLE DE INCIDENTES HUMANOS - CIH, 2011).

Nos hospitais existem as chamadas Comissões de Controle da Infecção Hospitalar - $\mathrm{CClH}$, formadas pelos próprios profissionais de saúde. Essas comissões são responsáveis pelo controle de fatores físicos, químicos e biológicos, evitando que patologias se proliferem. Porém, os profissionais de saúde que compõem essas equipes, com a função de gerir o ambiente hospitalar, não contam com abordagens específicas sobre gestão ambiental em suas formações. Apesar de discutido (o meio ambiente), formas de atuação sobre ele não são devidamente esclarecidas, sendo necessário ainda aprofundar o entendimento da relação existente entre a saúde e o ambiente.

Disso decorre uma série de dificuldades, como planos de gerenciamento de resíduos inadequados, monitoramento da qualidade da água deficiente, falta de padronização para os serviços de dedetização e controle de pragas, entre outros. Outro fator que contribui para que a gestão ambiental hospitalar não seja efetiva é que a equipe responsável por fazer gestão ambiental é a mesma equipe que presta assistência aos pacientes. Sendo a assistência o mais importante e primordial, a gestão acaba ficando de lado, não só contribuindo para ocorrência de infecções hospitalares, mas também para o acúmulo de pendências que podem levar a perda do Alvará Sanitário da Instalação.

Neste sentido, este trabalho se justifica em promover a gestão dos fatores ambientais intrínsecos à atividade, dos quais depende a eficácia do atendimento oferecido por essas Instituições.

Este trabalho propõe a utilização de metodologias de gestão para que os hospitais mantenham condições ambientais e sanitárias adequadas para a reabilitação das funções, capacidades e condições de saúde de seus usuários, no alcance da eficácia do atendimento oferecido por estas Instituições. A proposta envolve aplicações dos fundamentos e técnicas da Engenharia Ambiental, buscando a melhoria das condições de saneamento e saúde, por meio do levantamento e avaliação dos riscos inerentes ao ambiente hospitalar e formulação de propostas de controle.

\subsection{RISCOS NO AMBIENTE HOSPITALAR}

O ambiente, seja ele interno ou externo, pode conter fatores físicos, químicos e biológicos que podem funcionar como limitadores das condições de saúde de seus usuários. Estes fatores podem aparecer naturalmente no ambiente, mas seus efeitos podem ser mais perceptíveis quando ocorre desequilíbrio ou degradação das condições de salubridade ambiental. A exposição a estes fatores, geralmente involuntária, pode contribuir, ainda que indiretamente, para a ocorrência de doenças. (CARDOSO, 2005).

Para Camello et al. (2009), estes fatores podem ter comportamento variável sendo que a própria medida de presença ou ausência pode estar relacionada com a ocorrência de doenças.

Sendo assim, a Gestão Ambiental deve prever ferramentas adequadas para gestão dos fatores de risco ambientais, na qual deve estar incluído a sua identificação, diagnóstico, tratamento e monitoramento.

No contexto hospitalar, também se encontram esses fatores, sendo que tanto clientes quanto as equipes de trabalho estão expostos a eles. Em boas condições de higiene e saneamento, o risco de contaminação é relativamente baixo, sendo que a higienização e limpeza não eliminam esses fatores do ambiente, mas 
os colocam em situação de controle, de forma a diminuir ou neutralizar seu potencial de risco, ainda que temporariamente.

Fatores físicos como a arquitetura e engenharia das acomodações, instalações sanitárias, limpeza e conservação, sentido de fluxo de materiais e pessoas, acabamento de pisos e paredes podem facilitar ou dificultar a ocorrência de riscos ou de vetores.

Fatores químicos como os produtos utilizados pelas equipes de saúde, ou produtos usados para limpeza e desinfecção, podem oferecer riscos que decorrem tanto da utilização quanto do acondicionamento inadequados.

Os microrganismos também são agentes que podem causar agravos no ambiente hospitalar, onde é intenso o contato com as mais variadas classificações deles. (CAMELLO et al, 2009).

Os serviços médico-hospitalares são considerados fatores de risco de natureza dinâmica e são objeto de normatização e de vigilância sanitária, que é um campo que visa prevenir, diminuir e eliminar riscos e danos à saúde individual e coletiva, por meio do controle de produtos, tecnologia e serviços de interesse sanitário. Atua sobre os fatores de risco e atividade relacionados à saúde, ao ambiente físico e ao ambiente de trabalho, com base na aplicação de várias disciplinas do conhecimento, entre elas a epidemiologia e a engenharia (CAMELLO et al, 2009).

Os hospitais são locais onde se trata de diversas enfermidades, lida-se com produtos químicos e geram-se resíduos de classes diversas. O controle da sanidade ambiental pode significar a redução dos casos de infecção hospitalar, redução de acidentes do trabalho e doenças ocupacionais e o controle de passivos ambientais'.

Um dos problemas mais graves que podem surgir pela falta gestão ou pela gestão inadequada dos fatores ambientais é a infecção hospitalar. Segundo Hinrichsen e Pires (2009, p. 14):

\section{[...] A ocorrência de infecções hospitalares referentes ao ambiente físico engloba não só aspectos vinculados à arquitetura, mas também a engenharia de instalações, serviços de limpeza e conservação, constituindo apenas uma parte de todo este universo. Há necessidade de uma abordagem multidisciplinar capaz de controlar e prevenir os processos infecciosos nosocomiais. ${ }^{2}$}

A infecção hospitalar pode ser evitada por meio de gestos simples e cotidianos, como por exemplo, a lavagem das mãos. Para Lira et al (2009), a lavagem das mãos é prática prioritária e pode reduzir consideravelmente os casos de infecções. Porém, apesar de um ato simples, continua sendo pouco praticado.

Sendo assim, é necessário conhecer a rotina das equipes de trabalho e identificar se as boas práticas de higiene têm sido realizadas e, se não estiverem, os motivos que levam a isso, para então propor ações que possam motivar as equipes.

A gestão ambiental hospitalar deve prever a gestão de resíduos, vigilância sanitária e epidemiológica (principalmente na conservação e limpeza, controle de vetores e na lavanderia), adequações ambientais, treinamento e conscientização das equipes de trabalho, controle de riscos ocupacionais, biossegurança e controle da qualidade da água.

Quanto ao resíduo hospitalar, segundo Nogueira (1999), este necessita de cuidados que vão desde a classificação e segregação até a disposição final. O acondicionamento em sacos é feito por tipo de resíduo e diferenciação de cor. Para Hinrichsen et al (2009), o potencial de risco se refere a existência de materiais biológicos, produtos químicos, objetos perfurocortantes e lixo radioativo.

O hospital, visto como ambiente de trabalho necessita de avaliação e monitoramento, pois também existem riscos aos quais as equipes de trabalho estão expostas. É necessário compreender sempre as relações entre ambiente, saúde e trabalho. As equipes de saúde, as equipes da lavanderia, as equipes da cozinha e preparo de alimentos e as equipes de conservação e limpeza estão expostas, muitas vezes em longos períodos e com maior frequência, aos mesmos agentes de risco que afetam aos pacientes e que degradam a qualidade ambiental. Os programas de educação e capacitação, a gestão de riscos ambientais e as adequações no ambiente podem minimizar, ou ainda controlar, as ocorrências de acidentes ou contaminações, baseando-se principalmente em medidas de prevenção.

\footnotetext{
1 Por passivo ambiental, entende-se os custos e responsabilidades decorrentes de modificações ambientais para adequações a requisitos legais e compensação por danos causados a terceiros decorrentes de fatores ambientais intrínsecos a atividade (MAZZINI, 2004).

2 Nosocomial é a doença que se adquire em ambiente hospitalar, segundo Jacobs e Jacobs (2006).
} 
Se as condições do meio podem favorecer a ocorrência de patógenos, é fundamental o serviço de limpeza e conservação para a manutenção de níveis adequados de salubridade e, segundo Pedrosa e Macedo (1999), para preparar o ambiente para as atividades hospitalares.

O uso correto de saneantes e a manutenção de rotinas de limpeza e desinfecção são favorecidos quando são realizados programas de educação e esclarecimento quanto aos riscos existentes, criando a motivação para adequações nos procedimentos visando a biossegurança.

A biossegurança consiste no conjunto de ações de prevenção, minimização ou eliminação de riscos inerentes às atividades que possam comprometer a saúde das pessoas, do ambiente ou mesmo a qualidade do trabalho desenvolvido (HINRICHSEN, 2009).

Outro controle que deve ser realizado em hospitais é na lavanderia. Para Santos (1999), a lavanderia hospitalar deve transformar a roupa suja e contaminada em roupa limpa, sendo que ela não precisa estar estéril ao final da lavagem, mas livre da quantidade de microrganismos capaz de causar doenças. Quando a lavagem não é adequada, tanto os usuários da rouparia quanto as equipes da lavanderia ficam expostos ao risco de contaminação por patógenos.

O controle de vetores é fator primordial para a promoção da saúde, sendo auxiliar na medicina preventiva. No ambiente hospitalar é dado maior importância ao controle de artrópodes (insetos e aracnídeos) e roedores, que, com maior frequência, veiculam agentes infecciosos. (QUIRINO, 1999).

O controle da qualidade microbiológica da água também deve ser realizado, pois há doenças que podem ser transmitidas pela água de má qualidade. Segundo Vieira (1999), os enfermos, os lactentes ${ }^{3}$ e as crianças

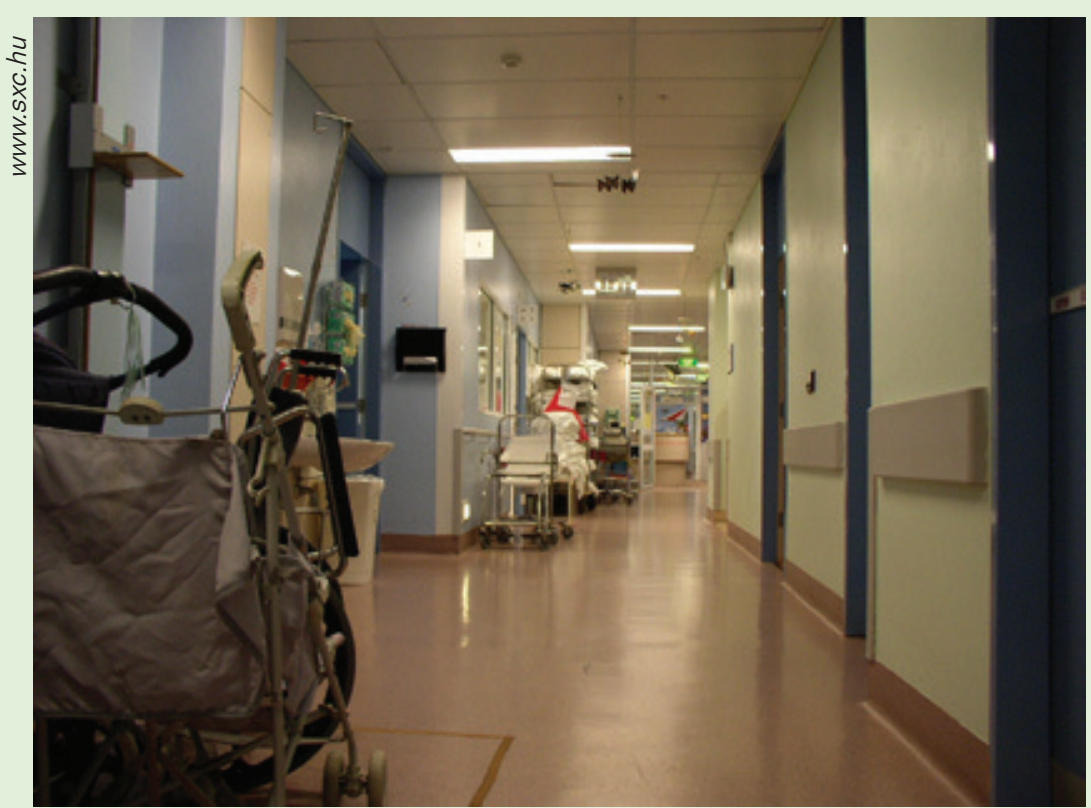
são mais vulneráveis. Uma das ações de monitoramento é a coleta periódica de amostras de água para análise de parâmetros.

Diante do exposto, a gestão em saúde do ambiente hospitalar requer interdisciplinaridade, devido à complexidade dos fatores envolvidos. A Engenharia Ambiental, que visa o controle do ambiente e melhoria da qualidade de vida das pessoas (MAZZINI, 2004), também pode ser aplicada neste contexto, participando da análise e gestão de todos os fatores apresentados.

\subsection{COMISSÕES DE CONTROLE DA INFECÇÃO HOSPITALAR}

As Comissões de Controle de Infecção Hospitalar $(\mathrm{CClH})$ são comissões criadas conforme determinação da Portaria Ministerial 2.616 de 12 de maio de 1998, aplicável à toda instituição de assistência em saúde.

A formação de uma $\mathrm{CClH}$ tem como objetivo básico o equacionamento dos problemas inerentes ao desenvolvimento das infecções institucionais, partindo da identificação e reconhecimento de suas causas, levando a definição de diretrizes para as ações de controle das infecções, avaliando e ratificando o programa de controle das infecções institucionais, implantando medidas de níveis aceitáveis para a segurança dos usuários.

A CCIH deve ser uma comissão permanente, atuando como órgão de acessoria, com autonomia plena para decidir sobre assuntos e traçar metas que, direta ou indiretamente, estiverem relacionadas com a prevenção e controle das infecções.

3 Lactente é a criança que ainda mama. (Jacobs; Jacobs, 2006). 
Nesse sentido, compete às $\mathrm{CClH}$ : a) elaborar, implementar e manter o Programa de Controle de Infecções; b) implantar sistema de vigilância epidemiológica, adequar as rotinas técnico-operacionais; c) oferecer capacitação e treinamento aos colaboradores; d) controlar o uso de antimicrobianos, germicidas, e materiais; realizar investigações epidemiológicas; e) desenvolver índices de monitoramento e previsão de casos de infecção hospitalar; implantar medidas imediatas de controle de focos de infecção e medidas de prevenção à longo prazo; f) elaborar e divulgar relatórios sobre a situação da instalação; g) elaborar e implantar normas internas operacionais e de conduta; h) notificar ao serviço de vigilância os casos de infecção verificados; i) deliberar sobre a gestão de resíduos, qualidade de água, adequações ambientais, controle de pragas e vetores, condutas de limpeza e conservação e esterilização/ desinfecção de ambientes, materiais e equipamentos (BRASIL, 1998).

Quanto à composição, deve conter membros consultores e executores. No grupo de consultores, deve haver, no mínimo, um representante da equipe médica, um representante da administração, um representante da farmácia, um representante do laboratório de microbiologia e um representante da enfermagem. A equipe de executores é formada de técnicos, cuja quantidade é estipulada conforme a quantidade de leitos existente (sendo 2 para cada 200 leitos). A Portaria recomenda que um seja preferencialmente da área de enfermagem. As cargas horárias diárias para controle de infecção variam entre 4 e 6 horas. Contudo, não há restrição quanto à participação de outros profissionais que possam colaborar para o trabalho.

Outra responsabilidade da $\mathrm{CCIH}$ é a criação de indicadores de vigilância epidemiológica. A Portaria 2.616 recomenda que sejam estabelecidos ao menos os indicadores mais importantes, a saber: a) taxa de infecção hospitalar; b) taxa de pacientes com infecção hospitalar; c) distribuição percentual das infecções hospitalares por topografia no paciente; d) taxa de infecções hospitalares por procedimento (taxa de pneumonia após uso de respirador ou taxa de infecção urinária após cateterismo vesical, por exemplo); e) taxa de infecção do sítio cirúrgico, por procedimento e por cirurgião; f) freqüência de infecções hospitalares por microrganismos ou por etiologia; g) coeficiente de sensibilidade a antimicrobianos; h) indicadores de uso de antimicrobianos; i) taxa de letalidade associada a infecção hospitalar (BRASIL, 1998).

Porém, nem sempre os indicadores são alimentados devidamente, ou os casos de infecção são devidamente reconhecidos como tal. Ainda, nem sempre são tomadas as medidas preventivas como lavagem das mãos e higienização, que poderiam evitar ou pelo menos diminuir a incidência de casos. Apesar dos planos, normas e padronizações, a realidade das atividades é bastante diferente dos planos.

\section{METODOLOGIA DE TRABALHO}

Esta é uma pesquisa descritiva exploratória e se baseia em uma proposta acadêmica de análise e gestão de riscos do ambiente hospitalar, em especial a infecção hospitalar. O trabalho foi desenvolvido por um grupo de acadêmicos do curso de Engenharia Ambiental da Universidade de Uberaba, em Uberaba/MG, orientado pelo docente da disciplina Análise de Riscos.

Durante reuniões do grupo, realizadas na Universidade, definiu-se a metodologia a ser utilizada para o desenvolvimento do trabalho.

Definiu-se que seriam visitados três hospitais de médio porte da região do Triângulo Mineiro, que prestassem atendimento público e particular, com base no raciocínio que o levantamento realizado em três instituições ofereceria dados suficientes para a efetivação da proposta deste trabalho.

A escolha dos hospitais foi aleatória. O aceite para a realização das atividades foi evidenciado pela assinatura de termo de consentimento por parte das Instituições, na garantia de sua não identificação.

Não foi utilizado questionário para a coleta de dados. Os dados foram coletados por meio de relato dos gestores das Instituições com base em temas de análise pré-definidos, que estão relacionados à infecção hospitalar: a) monitoramento da qualidade da água; b) controle de vetores; c) gerenciamento de resíduos de serviços de saúde; d) vigilância sanitária e; e) treinamento e conscientização dos colaboradores. Os dados relatados foram transcritos manualmente.

Após as visitas, os dados foram analisados qualitativamente por meio de ferramentas de gestão de riscos e gestão da qualidade como a Análise de Operabilidade de Perigos - HAZOP, Brainstorming e Diagrama de Causa e Efeito, apontando os riscos mais emergentes em cada uma das Instituições visitadas, para os quais o grupo definiu propostas de gestão baseadas na metodologia do Plano de Ação - 5W2H e alinhadas à metodologia ISO 31000 (Norma ISO para Análise de Riscos). 
Neste estudo, as ferramentas são apresentadas em sete análises:

- Folha de Verificação - utilizada para listar todos os problemas ocorridos no processo e quantificá-los, antes que se aplique o Diagrama de Causa e Efeito ou a Curva ABC na priorização identificação dos problemas. As folhas de verificação são tabelas ou planilhas para agilizar as coletas de dados, mas não possuem padrões; assim, cada organização deverá desenvolver seus formulários de dados conforme suas necessidades.

- Curva $A B C$ ou Princípio de Pareto - será aplicado na identificação e seleção das principais causas que afetam a área analisada. O Princípio de Pareto revela que, $80 \%$ dos problemas estão relacionados a apenas $20 \%$ das causas. Graficamente, o Princípio de Pareto é representado por barras em ordem decrescente, para facilitar a visualização das prioridades, e uma curva com percentual acumulado, indicando os produtos/processos que deverão ser priorizados.

- Diagrama de Causa e Efeito ou Diagrama de Ishikawa - aplicado na identificação de "quais e como" os problemas relacionam-se ao analisar suas causas e efeitos pelos fatores: materiais, mão de obra, máquinas e métodos de trabalho e meio ambiente.

- Fluxograma - por meio de símbolos padronizados, os fluxogramas formação os caminhos representativos das etapas, que permitirão identificar nos "percursos" as causas que ocasionam falhas e gargalos.

- Histograma - é uma forma de representação gráfica, expressa normalmente por barras, que permitirá analisar grandes quantidades de dados pela frequência que eles ocorrem. Sua construção permitirá identificar quais dados tendem a indicar as possíveis causas reais do problema analisado por probabilidades.

- Diagrama de Dispersão - aplicado como uma forma de representação para analisar dois grupos de dados (coletados aos pares) e descobrir a relação que os envolve - variáveis causa e efeito.

- Cartas de Controle - são aplicadas para avaliar a variabilidade dos processos entre pontos estatisticamente determinados (faixas limites de tolerâncias - superior, média e inferior) por variáveis ou atributos. As faixas apontarão quais as variações que estão sob controle correspondem às causas inerentes ao processo.

- Estudos de Riscos e Operabilidade (HAZOP - Hazard and Operability Study) - método que permitirá a equipe identificar os riscos e os problemas de operabilidade. O HAZOP utiliza de palavras-guia que e consiste na realização de várias reuniões, durante as quais a equipe foca os pontos específicos da pesquisa, os chamados "nós de estudo" para alocar idéias e soluções para os riscos identificados. As palavras-guia são palavras simples, utilizadas para qualificar ou quantificar a intenção de modo a guiar e estimular o processo de criatividade e, assim, descobrir desvios.

- 5W2H - utiliza das siglas da língua inglesa e sua definição é: What, Who, When, Where, Why, How e How Much. Funciona nesta pesquisa como um mapeamento das atividades, estabelecendo o que será feito, quem fará o quê, em qual período de tempo, em qual área e todos os motivos pelos quais esta atividade deve ser feita. E, de forma útil, esta ferramenta tende a eliminar por completo qualquer dúvida que possa surgir sobre um processo ou atividade após o início das tarefas.

Serão apresentados a seguir os resultados da identificação e avaliação dos riscos utilizando as ferramentas citadas acima. Para evidenciar a aplicabilidade de cada ferramenta para análises aplicadas ao contexto hospitalar, sem que a leitura se pareça exaustiva, serão apresentados os resultados de uma ferramenta por hospital.

\section{IDENTIFICAÇÃO E AVALIAÇÃO DOS RISCOS}

No Hospital A foi verificado que: não havia CCIH formada, não havia Plano de Gerenciamento de Resíduos, não havia metodologia de controle da qualidade da água e não havia padronização na contratação dos serviços de controle de pragas.

No Hospital $\mathrm{B}$ já havia $\mathrm{CCIH}$ formada; o controle de qualidade da água estava deficiente quanto à rastreabilidade dos documentos, análise e monitoramento dos resultados e controle de datas de coleta; não havia, no período, empresa responsável por dedetização e controle de pragas, nem padronização dos critérios para contratação dos serviços; as equipes estavam em fase de treinamento para implantação da 
coleta seletiva; e o Plano de Gerenciamento de Resíduos de Serviços de Saúde, apesar de implantado, não continha indicadores de monitoramento e faltava adequação a alguns critérios da Resolução Anvisa 306, que estabelece regras para o gerenciamento de resíduos de serviços de saúde.

No Hospital C, o Plano de Gerenciamento de Resíduos de Serviços de Saúde necessitava de adequações, os serviços de dedetização e controle de pragas estava deficiente e havia casos de reclamações de pacientes quanto ao gosto da água do bebedouro, cujos filtros não tinham regularidade de troca nem regularidade de coleta de amostras para análise.

Em ambos os hospitais, não se teve acesso à dados de infecção hospitalar. Em ambos não havia laboratório de microbiologia.

Foram escolhidas três ferramentas para análise dos riscos: Braistorming, HAZOP e Diagrama de Causa e Efeito (também conhecido por Diagrama de Ishikawa). O problema em análise será a Infecção Hospitalar.

\subsection{Brainstorming}

Após as visitas, o grupo se reuniu para o Brainstorming sobre causas dos problemas verificados que podem levar a casos de infecção hospitalar. Do Brainstorming realizado, foram relacionadas as seguintes hipóteses (QUADRO 1), com relação ao Hospital B:

\section{Quadro 1 - Brainstorming realizado para o Hospital B}

\begin{tabular}{|c|c|c|}
\hline Plano de Gerenciamento de Resíduos & Controle de Qualidade da Água & Dedetização e Controle de Valores \\
\hline $\begin{array}{l}\text { Falta de conhecimento para elaboração } \\
\text { Falta de atualização } \\
\text { Falta de discussão por parte da CCIH } \\
\text { Conhecimento insuficiente da Res. } 306 \\
\text { Falta de cobrança por parte de fiscalização } \\
\text { Realização apenas para atendimento legal } \\
\text { Pouca participação das equipes na confecção do plano } \\
\text { Falta de leitura do plano } \\
\text { Falta de divulgação do plano } \\
\text { Falta de tempo das equipes } \\
\text { Falta de vontade das equipes } \\
\text { Pouca dedicação da CCIH } \\
\text { Excesso de carga de trabalho em assistência }\end{array}$ & $\begin{array}{l}\text { Falta de conhecimento para monitoramento } \\
\text { Pouco conhecimento para interpretação dos laudos } \\
\text { Falta de divulgação dos resultados } \\
\text { Custo das análises } \\
\text { Falta de tempo das equipes } \\
\text { Falta de vontade das equipes } \\
\text { Pouca dedicação da CCIH } \\
\text { Excesso de carga de trabalho em assistência } \\
\text { Falta de tabela para controle de datas } \\
\text { Falta de organização dos documentos } \\
\text { Falta de responsável por guarda dos documentos } \\
\text { Falta de local adequado }\end{array}$ & $\begin{array}{l}\text { Custo dos serviços } \\
\text { Falta de responsável para formalização do contrato } \\
\text { Falta de empresas interessadas } \\
\text { Falta de conhecimento dos requisitos para realização } \\
\text { dos serviços } \\
\text { Falta de formalização de contrato } \\
\text { Falta de padronização para contratação }\end{array}$ \\
\hline $\begin{array}{l}\text { Eleitos por principais: } \\
\text { Excesso de carga de trabalho em assistência; } \\
\text { Falta de conhecimento para elaboração e } \\
\text { Falta de atualização. }\end{array}$ & $\begin{array}{l}\text { Eleitos por principais: } \\
\text { Falta de conhecimento para monitoramento; } \\
\text { Falta de tempo das equipes e } \\
\text { Falta de tabela para controle de datas. }\end{array}$ & $\begin{array}{l}\text { Eleitos por principais: } \\
\text { Falta de padronização para contratação e } \\
\text { Falta de formalização de contrato }\end{array}$ \\
\hline
\end{tabular}

Fonte - Acervo dos autores (2011)

\subsection{HAZOP}

Será apresentado a seguir o Hazop aplicado ao Hospital A, onde foi verificado que: não havia $\mathrm{CCIH}$ formada, não havia Plano de Gerenciamento de Resíduos, não havia metodologia de controle da qualidade da água e não havia padronização na contratação dos serviços de controle de pragas (Figura 1). 
Figura 1 - Matriz HAZOP

\begin{tabular}{|c|c|c|c|c|}
\hline \multicolumn{5}{|c|}{ UNIDADE DE PROCESSO: Hospital A } \\
\hline \multicolumn{5}{|c|}{ NÓ DE ESTUDO: 01 PARÂMETRO DE PROCESSO : Fluxo de Controle de Infecção Hospitalar } \\
\hline PALAVRA-GUIA & DESVIO & CAUSAS & CONSEQUÊNCIA & AÇÕES SUGERIDAS \\
\hline Nenhum & $\begin{array}{l}\text { Ausência de } \\
\mathrm{CCIH}\end{array}$ & $\begin{array}{l}\text { (1) ausência de interessados em } \\
\text { participar da comissão } \\
\text { (2) ausência de profissionias } \\
\text { habilitados } \\
\text { (3) carga horária disponível dos } \\
\text { profissionais }\end{array}$ & $\begin{array}{l}\text { (1) Redução ou bloqueio de recursos } \\
\text { repassados pelo SUS; } \\
\text { (2) Pendências diante da Vigilância Sanitária; } \\
\text { (3) Possibilidade de perda do Alvará Sanitário; } \\
\text { (4) Maior propensão a ocorrência da Infecção } \\
\text { Hospitalar. }\end{array}$ & $\begin{array}{l}\text { (1) Implantação do } \mathrm{CCIH} \text {; } \\
\text { (2) Elaboração de Cronograma de } \\
\text { reuniões; } \\
\text { (3) Formalização da CCIH junto aos } \\
\text { órgãos regulamentadores de saúde } \\
\text { (4) Treinamento das equipes de trabalho; } \\
\text { (5) Contratação de Engenheiro Ambiental. }\end{array}$ \\
\hline Nenhum & $\begin{array}{l}\text { Ausência de } \\
\text { PGRSS }\end{array}$ & $\begin{array}{l}\text { (1) carga de trabalho dos } \\
\text { profissionais } \\
\text { (2) ausência de profissionias } \\
\text { habilitados } \\
\text { (3) Interesse da administração }\end{array}$ & $\begin{array}{l}\text { (1) Redução ou bloqueio de recursos } \\
\text { repassados pelo SUS; } \\
\text { (2) Pendências diante da Vigilância Sanitária; } \\
\text { (3) Possibilidade de perda do Alvará Sanitário; } \\
\text { (4) Maior propensão a ocorrência da Infecção } \\
\text { Hospitalar; } \\
\text { (5) Proliferação de vetores. }\end{array}$ & $\begin{array}{l}\text { (1) Elaboração do PGRSS; } \\
\text { (2) Elaboração de Plano de Adequações; } \\
\text { (3) Treinamento e conscientização das } \\
\text { equipes quanto à metodologias de } \\
\text { gerenciamento de resíduos; } \\
\text { (4) Formalização das adequações junto à } \\
\text { Vigilância Sanitária; } \\
\text { (5) Treinamento das equipes de trabalho. }\end{array}$ \\
\hline Nenhum & $\begin{array}{l}\text { Controle de } \\
\text { Qualidade de } \\
\text { Água }\end{array}$ & $\begin{array}{l}\text { (1) carga de trabalho dos } \\
\text { profissionais } \\
\text { (2) ausência de profissionias } \\
\text { habilitados } \\
\text { (3) Interesse da administração }\end{array}$ & $\begin{array}{l}\text { (1) Doenças de veiculação hídrica; } \\
\text { (2) Água em qualidade inadequada para os } \\
\text { procedimentos médico-hospitalares; } \\
\text { (3) Perda de qualidade e segurança nos } \\
\text { serviços de nutrição; } \\
\text { (4) Redução ou bloqueio de recursos } \\
\text { repassados pelo SUS; } \\
\text { (5) Pendências diante da Vigilância Sanitária; } \\
\text { (6) Possibilidade de perda do Alvará Sanitário; }\end{array}$ & $\begin{array}{l}\text { (1) Elaborar planilha para o controle de } \\
\text { datas de coleta de amostras; } \\
\text { (2) Treinamento das equipes de trabalho } \\
\text { em análises de laudos; } \\
\text { (3) Contratação de Engenheiro Ambiental; } \\
\text { (4) Formalização de contrato para } \\
\text { realização de coletas }\end{array}$ \\
\hline Nenhum & $\begin{array}{l}\text { Menor } \\
\text { padronização } \\
\text { do Controle de } \\
\text { Pragas }\end{array}$ & $\begin{array}{l}\text { (1) Desconhecimentos fod ítens } \\
\text { normativos sobre controle de } \\
\text { pragas; } \\
\text { (2) Interesse da administração }\end{array}$ & $\begin{array}{l}\text { (1) Proliferação de doenças transmitidas por } \\
\text { vetores; } \\
\text { (2) Contaminação cruzada em alimentos; } \\
\text { (1) Redução ou bloqueio de recursos } \\
\text { repassados pelo SUS; } \\
\text { (2) Pendências diante da Vigilância Sanitária; } \\
\text { (3) Possibilidade de perda do Alvará Sanitário; }\end{array}$ & $\begin{array}{l}\text { (1) Elaborar planilha para o controle de } \\
\text { datas de dedetização; } \\
\text { (2) Contratação de Engenheiro Ambiental; } \\
\text { (3) Formalização de contrato para } \\
\text { realização do serviço. }\end{array}$ \\
\hline
\end{tabular}

Fonte - Acervo dos autores (2011)

\subsection{Diagrama de Causa e Efeito}

Será apresentado o Diagrama confeccionado ao hospital C, para relacionar os problemas verificados como causas da Infecção Hospitalar e levantar as causas fundamentais (Figura 2):

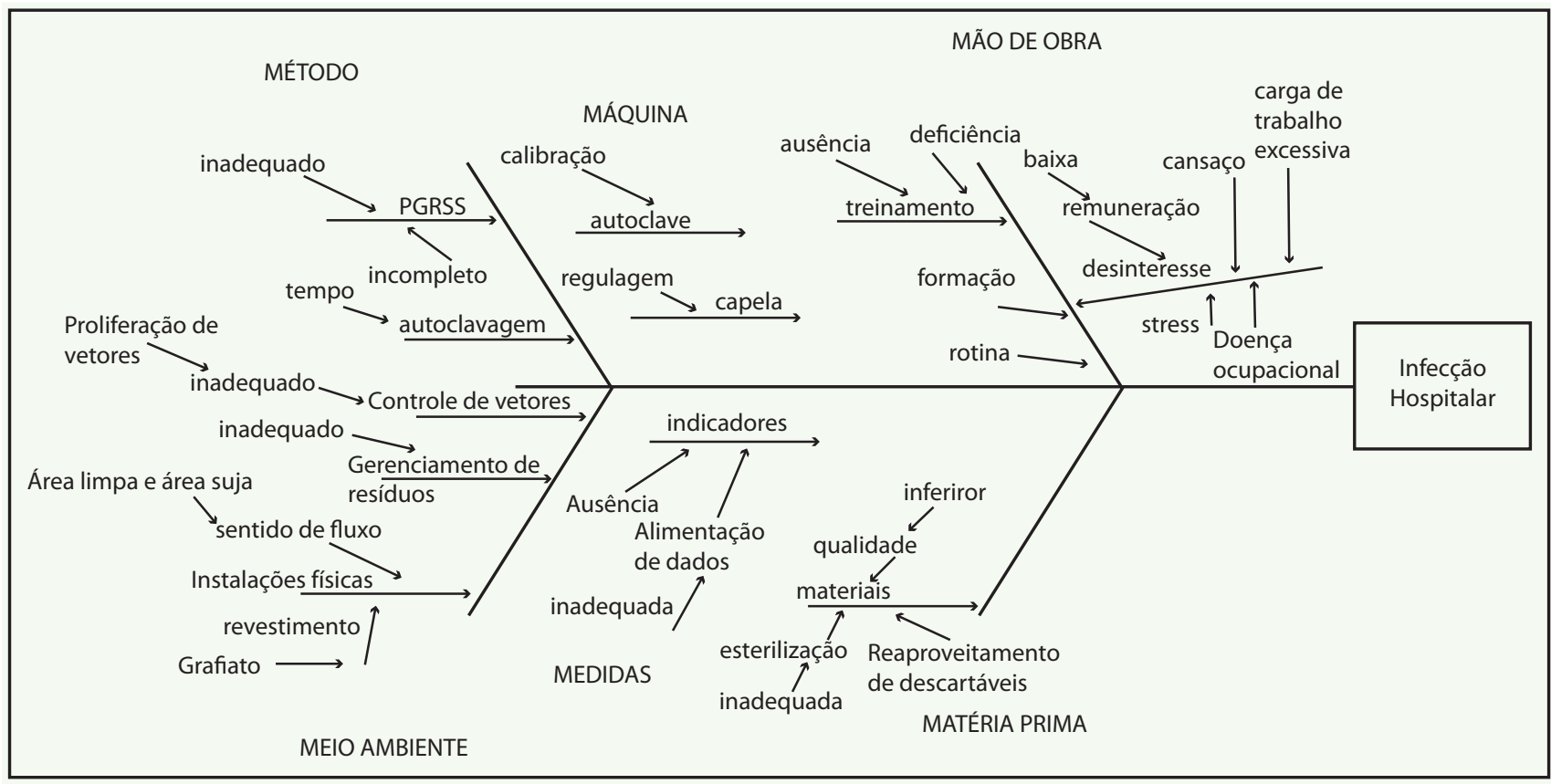

Fonte - Acervo dos autores (2011). 


\subsection{Priorização e adoção de medidas de controle}

Após o levantamento e diagnóstico das condições das 03 Instituições, se faz necessário o controle, por meio da minimização ou mitigação das causas dos problemas verificados.

A seguir são apresentadas alternativas viáveis ambiental e financeiramente. Para a formulação das propostas foram priorizadas as ações referentes a gerenciamento de resíduos, controle de vetores e qualidade da água que, independente do cenário ou da instalação, se aplicam a qualquer um deles.

As medidas de controle foram estipuladas utilizando a ferramenta Plano de Ação - 5W2H (FIGURA 3).

Tabela 1 - Utilização do Plano de Ação (Parte I)

\begin{tabular}{|c|c|c|c|c|}
\hline Item & O quê & Por quê & Quando & Onde \\
\hline I & $\begin{array}{l}\text { Elaboração ou revisão do } \\
\text { Plano de Gerenciamento de } \\
\text { Resíduos de Serviços de } \\
\text { Saúde - PGRSS }\end{array}$ & $\begin{array}{l}\text { Atendimento a requisitos } \\
\text { legais e prevenção de } \\
\text { infecção hospitalar }\end{array}$ & Prazo de 20 dias & $\begin{array}{l}\text { Nas dependências } \\
\text { do hospital }\end{array}$ \\
\hline ॥ & Implantar coleta seletiva & $\begin{array}{l}\text { Para se adequar às } \\
\text { recomendações do PGRSS }\end{array}$ & $\begin{array}{l}\text { No prazo de } 120 \\
\text { dias após aprovação } \\
\text { final do PGRSS }\end{array}$ & $\begin{array}{l}\text { Nas dependências } \\
\text { do hospital }\end{array}$ \\
\hline III & $\begin{array}{l}\text { Implantar Procedimento } \\
\text { Operacional Padrão para } \\
\text { contratação e realização dos } \\
\text { serviços de controle de pragas } \\
\text { e vetores }\end{array}$ & $\begin{array}{l}\text { Para atender a requisitos } \\
\text { legais e evitar infecção } \\
\text { hospitalar }\end{array}$ & Prazo de 15 dias & $\begin{array}{l}\text { Em todas as } \\
\text { dependências do } \\
\text { hospital }\end{array}$ \\
\hline IV & $\begin{array}{l}\text { Contratação de empresa } \\
\text { especializada para controle } \\
\text { de pragas }\end{array}$ & $\begin{array}{l}\text { Para atender ao } \\
\text { Procedimento Operacional } \\
\text { Padrão criado }\end{array}$ & $\begin{array}{l}\text { Prazo de } 30 \text { dias } \\
\text { após aprovação do } \\
\text { Procedimento }\end{array}$ & No mercado local \\
\hline V & $\begin{array}{l}\text { Implementação de tabela para } \\
\text { controle de datas de coleta de } \\
\text { amostra para análise físico- } \\
\text { química, bacteriológica e de } \\
\text { radioatividade da água }\end{array}$ & $\begin{array}{l}\text { Para garantir a regularidade } \\
\text { das análises de qualidade da } \\
\text { água utilizada }\end{array}$ & Prazo de 10 dias & $\begin{array}{l}\text { Programa Excel ou } \\
\text { similar }\end{array}$ \\
\hline VI & $\begin{array}{l}\text { Contratação de empresa } \\
\text { especializada para análise } \\
\text { de água }\end{array}$ & $\begin{array}{l}\text { Para fazer monitoramento } \\
\text { da qualidade da água }\end{array}$ & Prazo de 30 dias & No mercado local \\
\hline VII & $\begin{array}{l}\text { Realização de treinamento } \\
\text { sobre higienização das mãos e } \\
\text { de materiais }\end{array}$ & $\begin{array}{l}\text { Rapa reduzir em } 50 \% \text { a } \\
\text { chance de ocorrência } \\
\text { de infecção hospitalar } \\
\text { (probabilidade aceita } \\
\text { cientificamente) }\end{array}$ & semestralmente & $\begin{array}{l}\text { Na sala de } \\
\text { treinamentos }\end{array}$ \\
\hline VIII & $\begin{array}{l}\text { Desenvolver índices de } \\
\text { monitoramento e previsão de } \\
\text { casos de infecção hospitalar }\end{array}$ & $\begin{array}{l}\text { Para monitorar o } \\
\text { desempenho da instalação }\end{array}$ & $\begin{array}{l}\text { Prazo de } 10 \text { dias para } \\
\text { elaboração e coletas } \\
\text { de dados inicial. } \\
\text { Atualização mensal. }\end{array}$ & $\begin{array}{l}\text { Área } \\
\text { administrativa }\end{array}$ \\
\hline IX & $\begin{array}{l}\text { Levantar os custos } \\
\text { decorrentes de infecção e } \\
\text { gastos com prevenção }\end{array}$ & $\begin{array}{l}\text { Para visualizar o custo } \\
\text { benefício de programa de } \\
\text { prevenção }\end{array}$ & $\begin{array}{l}\text { Prazo de } 30 \text { dias } \\
\text { Atualização anual }\end{array}$ & $\begin{array}{l}\text { Área } \\
\text { administrativa }\end{array}$ \\
\hline
\end{tabular}

Fonte - Acervo dos autores (2011). 
Tabela 2 - Utilização do Plano de Ação (Parte II)

\begin{tabular}{|c|c|c|c|}
\hline Item & Quem & Como & Quanto Custa \\
\hline \multirow{6}{*}{ I } & \multirow{6}{*}{$\mathrm{CClH}$} & Levantamento de todas as atividades & $\mathrm{R} \$ 50,00$ \\
\hline & & Levantamento dos resíduos por atividade & $\begin{array}{l}\text { O custo da mão de obra } \\
\text { já faz parte da folha de } \\
\text { pagamento. }\end{array}$ \\
\hline & & $\begin{array}{l}\text { Classificação e quantificação de cada tipo de resíduo } \\
\text { por atividade }\end{array}$ & \\
\hline & & $\begin{array}{l}\text { Identificação dos procedimentos de segregação, } \\
\text { coleta, transporte, tratamento e disposição final }\end{array}$ & \\
\hline & & Elaboração de Plano de adequações & \\
\hline & & Estipulação de indicadores de monitoramento & \\
\hline \multirow{4}{*}{ II } & \multirow{4}{*}{$\mathrm{CCIH}$} & $\begin{array}{l}\text { Aquisição de lixeiras diferenciadas por cor para cada } \\
\text { setor do hospital; }\end{array}$ & \multirow{4}{*}{$\mathrm{R} \$ 12.000,00$} \\
\hline & & $\begin{array}{l}\text { Realização de treinamento de com todos os } \\
\text { colaboradores quanto à coleta seletiva; }\end{array}$ & \\
\hline & & $\begin{array}{l}\text { Revisão dos Procedimentos Operacionais Padrão } \\
\text { para incluir a coleta seletiva; }\end{array}$ & \\
\hline & & Instalação das lixeiras adquiridas. & \\
\hline \multirow[b]{2}{*}{ III } & \multirow[b]{2}{*}{$\begin{array}{l}\text { Membro designado } \\
\text { da CCIH }\end{array}$} & Consulta a requisitos legais pertinentes a atividade; & $R \$ 50,00$ \\
\hline & & $\begin{array}{l}\text { Pesquisa dos locais que devem ser dedetizados e } \\
\text { definição de como e com que periodicidade devem o ser. }\end{array}$ & $\begin{array}{l}\text { Os custos de mão de obra } \\
\text { já estão previstos a folha } \\
\text { de pagamento }\end{array}$ \\
\hline \multirow[b]{2}{*}{ IV } & \multirow{2}{*}{$\begin{array}{l}\text { Membro designado } \\
\text { da CCIH }\end{array}$} & Pesquisa de preço e cotação de mercado & \multirow[b]{2}{*}{$R \$$ 400,00/mês } \\
\hline & & $\begin{array}{l}\text { Formalização do certame com a proponente } \\
\text { vencedora }\end{array}$ & \\
\hline V & $\begin{array}{l}\text { Membro designado } \\
\text { da CCIH }\end{array}$ & $\begin{array}{l}\text { Criando planilha com preenchimento automático } \\
\text { da próxima data de coleta de acordo com a } \\
\text { periodicidade definida. A planilha deve sinalizar por } \\
\text { cor a proximidade da data ou expiração do prazo. }\end{array}$ & Sem custo adicional \\
\hline \multirow{2}{*}{$\mathrm{VI}$} & \multirow{2}{*}{$\begin{array}{l}\text { Membro designado } \\
\text { da CCIH }\end{array}$} & Pesquisa de preço e cotação de mercado & \multirow{2}{*}{ R\$ 700,00/mês } \\
\hline & & Formalização do certame com a proponente vencedora & \\
\hline VII & $\begin{array}{l}\text { Membro designado } \\
\text { da } \mathrm{CCIH}\end{array}$ & $\begin{array}{l}\text { Elaborar apresentação em datashow e elaborar } \\
\text { cartilha explicativa }\end{array}$ & Sem custo adicional \\
\hline VIII & Alta administração & $\begin{array}{l}\text { Implantando os índices recoemdados pela Portaria } \\
2.616 / 1998\end{array}$ & Sem custo adicional. \\
\hline IX & Alta administração & $\begin{array}{l}\text { Analisando os relatórios de ocorrência de infecção } \\
\text { hospitalar e analisando dados de aquisição de } \\
\text { materiais }\end{array}$ & Sem custo adicional. \\
\hline
\end{tabular}

Fonte - Acervo dos autores (2011). 


\section{4 - CONSIDERAÇÕES FINAIS}

A gestão dos riscos do ambiente hospitalar visa garantir a efetividade dos serviços prestados e evitar que pacientes e colaboradores contraiam patologias. Neste sentido, as ferramentas de análise de riscos e as ferramentas da qualidade se tornam importantes meios de prover informações e fazer monitoramento do desempenho das instalações.

Para a Engenharia Ambiental, aplicada à gestão hospitalar, se torna evidente que tanto o meio ambiente quanto a saúde são fatores complexos que precisam da atuação interdisciplinar para que, por meio da visão holística, seja possível abranger todos os aspectos que se correlacionam na determinação da saúde do meio e de seus usuários.

O trabalho realizado evidenciou a importância da interdisciplinaridade na gestão do ambiente e da saúde para a efetividade do serviço oferecido. Evidenciou ainda que medidas simples e de baixo custo podem ser aplicadas na prevenção dos riscos verificados.

As medidas de controle propostas são simples e de baixo custo, o que mostra que a efetividade da gestão pode ser alcançada mais com a sistematização das ações e monitoramento contínuo do que com complexas ações isoladas. A existência, nas Instituições Hospitalares, de profissional que possa se dedicar exclusivamente a Gestão Ambiental pode facilitar a implantação e continuidade das ações, bem como motivar as equipes para que participem também do processo de gestão por meio de suas ações cotidianas.

Por meio deste trabalho, evidencia-se que os fatores ambientais são importantes aspectos que devem ser considerados pela Gestão Hospitalar, inclusive com a manutenção de recursos financeiros e humanos para que sejam alcançados os níveis de salubridade e de desempenho almejados. 


\section{5 - REFERÊNCIAS}

BRASIL. Ministério da Saúde. Portaria n 2616, de 12 de maio de 1998. Dispõe sobre o Programa de Controle da Infecção Hospitalar. Disponível em < http://www.anvisa.gov.br/legis/portarias/2616_98. htm >. Acesso em 26 mar. 2011.

CARDOSO, Maria Rita Alves. Epidemiologia Ambiental. In: PHILLIPPI JR. (Ed.). Saneamento, Saúde e Ambiente. Barueri: Manole, 2005. cap. 4. p. 87-113. ISBN 85-204-2188-1. (Coleção Ambiental; 2).

CAMELLO, Thereza Cristina Ferreira et al. Gestão e Vigilância em Saúde Ambiental. Rio de Janeiro: Thex, 2009. 324 p. ISBN 978-85-7603-037-9.

CONTROLE DE INCIDENTES HUMANOS - CIH. Epidemiologia das Infecções Hospitalares. Disponível em: < http://www.cih.com.br/epidemiologia.htm\#volta>. Acesso em: 30 mai. 2011.

HINRICHSEN, Sylvia Lemos et al. Lixo hospitalar: gerenciamento dos resíduos sólidos dos serviços de saúde. In: B__. Biossegurança e controle de infecções: risco sanitário hospitalar. Rio de Janeiro: Guanabara Koogan, 2009. cap. 41. p. 230-248. ISBN 978-85-277-1517-1.

HINRICHSEN, Sylvia Lemos. Biossegurança: conceito e importância. In: Biossegurança e controle de infecções: risco sanitário hospitalar. Rio de Janeiro: Guanabara Koogan, 2009. cap. 1. p. 3-5. ISBN 978-85-277-1517-1.

HINRICHSEN, Sylvia Lemos; PIRES, Andrezza. Arquitetura hospitalar: orientações básicas para estabelecimentos de saúde. In: __. Biossegurança e controle de infecções: risco sanitário hospitalar. Rio de Janeiro: Guanabara Koogan, 2009. cap. 3. p. 14-23. ISBN 978-85-277-1517-1.

JACOBS, Karen; JACOBS, Laela (Ed.). Tradução de Vagner Raso. Dicionário de Terapia Ocupacional. São Paulo: Roca, 2006. 248 p. ISBN-10: 85-7241-615-3. ISBN-13: 978-85-7241-615-3. Título original: Quick reference dictionary for occupation therapy.

LIRA, Maria da Conceição et al. Higienização das mãos. In: HINRICHSEN, Sylvia Lemos. Biossegurança e controle de infecções: risco sanitário hospitalar. Rio de Janeiro: Guanabara Koogan, 2009. cap. 6. p. 38-49. ISBN 978-85-277-1517-1.

MAZZINI, Ana Luiza Dolabela de Amorim. Dicionário Educativo de Termos Ambientais. 2. ed. Belo Horizonte: A.L.D. Amorim Mazzini, 2004. 384 p. ISBN 85-903 655-1-4.

NOGUEIRA, José Mauro. Lixo hospitalar. In: COUTO, Renato Camargos; PEDROSA, Tânia Moreira Grillo; NOGUEIRA, José Mauro. Infecção hospitalar: epidemiologia, controle, gestão para a qualidade. 2. ed. [S.I.]: Medsi, 1999. cap. 10. p. 219-238. ISBN: 85-7199-196-0.

PEDROSA, Tânia Moreira Grillo; MACEDO, Rejane Maria. Serviço de limpeza. In: COUTO, Renato Camargos; PEDROSA, Tânia Moreira Grillo; NOGUEIRA, José Mauro. Infecção hospitalar: epidemiologia, controle, gestão para a qualidade. 2. ed. [S.I.]: Medsi, 1999. cap. 16. p. 323-327. ISBN: 85-7199-196-0.

QUIRINO, Nívea Eliza P. da Silva. Controle de Vetores. In: COUTO, Renato Camargos; PEDROSA, Tânia Moreira Grillo; NOGUEIRA, José Mauro. Infecção hospitalar: epidemiologia, controle, gestão para a qualidade. 2. ed. [S.I.]: Medsi, 1999. cap. 15. p. 317-323. ISBN: 85-7199-196-0.

SANTOS, Adélia Aparecida Marçal dos. A lavanderia e o controle de infecções hospitalares. In: COUTO, Renato Camargos; PEDROSA, Tânia Moreira Grillo; NOGUEIRA, José Mauro. Infecção hospitalar: epidemiologia, controle, gestão para a qualidade. 2. ed. [S.I.]: Medsi, 1999. cap. 11. p. 239-246. ISBN: 85-7199-196-0.

VIEIRA, Maria Berenice Cardoso Martins. Controle microbiológico da água. In: COUTO, Renato Camargos; PEDROSA, Tânia Moreira Grillo; NOGUEIRA, José Mauro. Infecção hospitalar: epidemiologia, controle, gestão para a qualidade. 2. ed. [S.I.]: Medsi, 1999. cap.38. p. 627-638. ISBN: 85-7199-196-0. 